\title{
ASPECTS OF FAULTY POSTURES OF THE SPINE IN FEMALE RHYTHMIC GYMNASTICS
}

\author{
Moraru Cristina - Elena ${ }^{1 *}$ \\ 1 "Alexandru Ioan Cuza" University, Carol Street 11, Iasi, 700506, Romania
}

Keywords: faulty posture, spine, rhythmic gymnastics

\begin{abstract}
Certain female dominated sports, such as rhythmic gymnastics, dancing and figure skating are characterized by an extreme range of motion of the spinal column. Athletes who practice such sports are more prone to injury of the vertebral column and to the abnormal development of the spinal column. The purpose of this paper was to assess the presence of lordotic or scoliotic attitudes among performance rhythmic gymnasts. Early identification of faulty postures can lead to the initiation of a recovery program meant to prevent their evolution. Within this study, we analyzed ten $4^{\text {th }}$ - category gymnasts aged between 8 and 10. We performed the measurements using the GPS 400Global Postural System. After measurements, we identified 10 gymnasts with lordotic postures and 8 of them with scoliotic and lordotic postures. As a general conclusion, we highlight the need to implement individual programs for the rehabilitation or correction of faulty postures, to be performed following specific trainings.
\end{abstract}

\section{Introduction}

The spine is a flexible, multisegmented column. Its functional role is to maintain stability and an upright position as well as providing mobility at the segmental level. The spine comprises a static, changeless element - the vertebral bodies - and an elastic mobile component - the three - joint complex, consisting of the intervertebral disc and the two posterior facet joints. Spine motion, stability, equilibrium and control of position are assumed by the antagonist action of the powerful flexor and extensor muscle groups. (Benoist, 2003, p. 86)

Spinal deformity represents a spectrum of disorders that commonly start in childhood or adolescence and may have ramifications of pain and deformity in adults. (D’Hemecourt, 2012, p. 25)

The high incidence of postural disorders in certain branches of sport, especially among adolescents has questioned the influence of sports activities on the posture of athletes. Deviations from correct posture, as a result of a specific sport or excessive training loads can be found in the works of many authors

\footnotetext{
*E-mail: gimcristinamoraru@yahoo.com, tel.0744.933.133
} 
(Grabara, \& Hadzik, 2009; Slawinska, Rozek, \& Ignasiak, 2006).

Some postural disorders are more common in certain sports fields, so it is assumed that the specific requirements of sport and training loads that occur during the execution of technical elements and prolonged repetition of these elements influenced the development of those postural disorders. Some female dominated sports, such as rhythmic gymnastics, figure skating and dancing are characterized by an extreme range of motion of the spinal column (Cirillo, \& Jackson, 1985; Micheli 1983; Sward, Hellstrom, \& Jacobsonn, 1990). Athletes who practice these types of sports have a higher risk of injury of the vertebral column and the abnormal development of the spinal column.

There is not a single sport nowadays where athletes do not have a disorder of the spinal column, whereas the level and the type of disorder depend on the nature of the sport. (Stošić et al., 2011, p. 375)

The capacity of performing in rhythmic gymnastics is very complex, since the gymnast is frequently facing dual-task situations: jumping, twisting or balance with starting or handling apparatus. Therefore, we cannot consider only the quantitative aspect, but also the quality of execution that depends directly on the level of coordination, technical mastery, and physical performance of the gymnasts. (Mkaouer et al., 2012, p. 75)

To combine many elements, a gymnast must have highly developed proprioception and motor control, obtained through neurological development, through years of practice. The neuromuscular system must coordinate movements, and very fine control is required. (Brooks, 2011)

The rhythmic gymnasts need an extreme flexibility and also strength skills to rapidly reach and fix the wide shapes of technical leaps required by International Code of Points. (Brooks, 2003, p. 23; Di Cagno, 2010, p. 1995).

There are many different body positions required in the sport of gymnastics that are relevant to back injury. Rhythmic gymnasts also train and compete in multiple events. In general, stress is placed on the spine via skills that require extreme levels of flexion and extension of the vertebral column not seen in other sports.

The casual observer will note that the sport of gymnastics requires a level of flexibility, conditioning, and complete body recruitment that is matched infrequently by other sports. (Kruse \& Lemmen, 2009, p. 20).

Low back pain is highly prevalent in gymnasts and multiple epidemiologic studies and reviews have documented this with prevalence ranging from 25\% to 85\%. For rhythmic gymnasts, one study published in 1999 showed reports low back pain in $86 \%$ of the gymnasts studied. (Hutchinson, 1999, p. 1686)

\section{Material and methods}

The development of an appropriate treatment plan depends on a thorough assessment of the musculoskeletal and neuromuscular systems that provide spinal stability followed by the proper prescription of therapeutic exercises to 
correct the identifiable deficits. A comprehensive rehabilitation program should include correction of muscle imbalances that are a result of flexibility, strength, endurance, and balance deficits. (Donatelli \& Dimond, 2012, p. 12)

Certain female dominated sports, such as rhythmic gymnastics, dancing and figure skating are characterized by an extreme range of motion of the spinal column; this region of the body is highly solicited. Some postural disorders are more common in certain sports fields, so it is assumed that the specific demands of sport and training loads that occur while executing technical elements and during prolonged repetition of these elements had an influence on the development of faulty postures. Athletes who practice such sports are more prone to injury of the vertebral column and to the abnormal development of the spinal column.

The purpose of this paper was to assess the presence of lordotic or scoliotic attitudes among performance rhythmic gymnasts.

Working hypothesis. Early identification of faulty postures in female rhythmic gymnasts can lead to the initiation of a recovery program meant to prevent their evolution.

Within this study, we analyzed ten $4^{\text {th }}$ - category gymnasts aged between 8 and 10. We performed the measurements using the GPS 400-Global Postural System.

\section{Results and Discussions}

After measurements, we identified 10 gymnasts with lordotic postures and 8 of them with scoliotic and lordotic postures.

Table 1 Functional diagnosis of female rhythmic gymnasts

\begin{tabular}{|c|c|c|}
\hline $\begin{array}{c}\text { N.P/ } \\
\text { Deviation }\end{array}$ & Functional diagnosis & $\begin{array}{c}\text { Recommenda } \\
\text { tions }\end{array}$ \\
\hline $\begin{array}{l}\text { V.L. } \\
\text { In frontal } \\
\text { plane }\end{array}$ & $\begin{array}{l}\text { Dextroconvex dorsolumbar scoliotic posture } \\
\text { - } \quad \text { Asymmetric shoulders and scapulae, left lower by } 4^{\circ} ; \\
\text { - } \quad \text { Asymmetric basin, left side higher by } 3^{\circ} ; \\
\text { - } \quad \text { Uneven waist triangles, more pronounced on the left side. }\end{array}$ & $\begin{array}{l}\text { We recommend } \\
\text { kinesiotherapy, } \\
\text { orthopaedic consult } \\
\text { and spine X-ray }\end{array}$ \\
\hline $\begin{array}{l}\text { In sagittal } \\
\text { plane }\end{array}$ & $\begin{array}{l}\text { Hyperlordotic posture with marked curvature } \\
\text { - } \quad \text { Head and neck slightly projected forwards; } \\
\text { - } \quad \text { Cervical arrow } 1.1 \mathrm{~cm} ; \\
\text { - } \quad \text { Lumbar arrow } 3.4 \mathrm{~cm} ; \\
\text { - } \quad \text { Barycentre deviation forwards, with greater support } \\
\text { on the front leg and with anteverted pelvis. }\end{array}$ & $\begin{array}{l}\text { We recommend } \\
\text { kinesiotherapy, } \\
\text { orthopaedic and } \\
\text { radiology consult }\end{array}$ \\
\hline $\begin{array}{l}\text { A.R. } \\
\text { In frontal } \\
\text { plane }\end{array}$ & $\begin{array}{l}\text { Slight tendency of dextroconvex dorsolumbar scoliotic posture } \\
-\quad \text { Asymmetric shoulders, left lower by } 3^{\circ} ; \\
-\quad \text { Asymmetric scapulae, left lower by } 3^{\circ} ; \\
-\quad \text { Slightly more pronounced waist triangle on the left; } \\
\text { - } \quad \text { Incorrect head position (towards the left and inclines } \\
\text { towards the right). }\end{array}$ & $\begin{array}{l}\text { We recommend } \\
\text { postural self- } \\
\text { control, active } \\
\text { posture correction, } \\
\text { back muscles } \\
\text { toning. }\end{array}$ \\
\hline In sagittal & Hyperlordotic posture & We recommend \\
\hline
\end{tabular}




\begin{tabular}{|c|c|c|}
\hline plane & $\begin{array}{l}\text { - Head and neck slightly projected forwards; } \\
\text { - Cervical arrow } 1.3 \mathrm{~cm} \text {; } \\
\text { - } \text { Lumbar arrow } 3.4 \mathrm{~cm} \text {; } \\
\text { - } \text { Anteverted pelvis, knees slightly hyperextended. }\end{array}$ & $\begin{array}{l}\text { kinesiotherapy to } \\
\text { correct posture, } \\
\text { abdominal muscles } \\
\text { toning and pelvis } \\
\text { position correction. }\end{array}$ \\
\hline $\begin{array}{l}\text { A.D. } \\
\text { In frontal } \\
\text { plane }\end{array}$ & $\begin{array}{l}\text { Slight dorsolumbar levoconvex scoliotic posture } \\
\text { - Asymmetric shoulders, the right lower by } 4^{\circ} \text {; } \\
\text { - Distance between ear lobe and acromioclavicular joint } \\
\text { greater by } 2 \mathrm{~cm} \text { on the right side; } \\
\text { - Uneven waist triangles, sharper and larger by } 1.9 \mathrm{~cm} \text { on } \\
\text { the right; } \\
\text { - Slightly higher pelvis, by } 1-2^{\circ} \text { on the right side }\end{array}$ & $\begin{array}{l}\text { We recommend } \\
\text { kinesiotherapy to } \\
\text { re-educate correct } \\
\text { posture in this } \\
\text { plane. }\end{array}$ \\
\hline $\begin{array}{l}\text { In sagittal } \\
\text { plane }\end{array}$ & $\begin{array}{l}\text { Cervicodorsal spine straightness and high lumbar } \\
\text { hyperlordosis } \\
\text { - Upper cervical and dorsal spine straightness; } \\
\text { - Cervical arrow } 0.8 \mathrm{~cm} \text {; } \\
\text { - Lumbar arrow } 4 \mathrm{~cm} \text {; } \\
\text { - Extended exaggerated lumbar curvature, also affecting } \\
\text { the dorsal area }\end{array}$ & $\begin{array}{l}\text { We recommend } \\
\text { kinesiotherapy for } \\
\text { postural } \\
\text { realignment, for } \\
\text { physiological } \\
\text { curvatures } \\
\text { correction, } \\
\text { orthopaedic and } \\
\text { radiology consult }\end{array}$ \\
\hline $\begin{array}{l}\text { L.M. } \\
\text { In frontal } \\
\text { plane }\end{array}$ & $\begin{array}{l}\text { Dextroconvex dorsolumbar scoliotic posture } \\
\text { - Asymmetric shoulders and scapulae, lower on the left } \\
\text { side; } \\
\text { - Uneven waist triangles, sharper on the left side; } \\
\text { - Asymmetric pelvis, higher on the left side. }\end{array}$ & $\begin{array}{l}\text { We recommend } \\
\text { kinesiotherapy }\end{array}$ \\
\hline $\begin{array}{l}\text { In sagittal } \\
\text { plane }\end{array}$ & $\begin{array}{l}\text { Hyperlordotic posture } \\
\text { - } \text { Head and neck slightly projected forwards; } \\
\text { - } \text { Cervical arrow } 2.4 \mathrm{~cm} ; \\
\text { - } \text { Lumbar arrow } 4.4 \mathrm{~cm} ; \\
\text { - } \text { Anteverted pelvis. }\end{array}$ & $\begin{array}{l}\text { We recommend } \\
\text { abdominal muscles } \\
\text { toning and dorsal } \\
\text { muscles toning }\end{array}$ \\
\hline $\begin{array}{l}\text { F.D. } \\
\text { In frontal } \\
\text { plane }\end{array}$ & $\begin{array}{l}\text { Levoconvex dorsal and dextroconvex lumbar scoliotic } \\
\text { posture } \\
\text { - Asymmetric shoulders and scapulae, right lower by } 2^{\circ} \text {; } \\
\text { - Asymmetric waist triangles in both size and shape, } \\
\text { sharper on the left side and slightly larger on the right; } \\
\text { - } \quad \text { Slightly asymmetric pelvis, higher on the left side. }\end{array}$ & $\begin{array}{l}\text { We recommend } \\
\text { posture correction } \\
\text { through } \\
\text { kinesiotherapy }\end{array}$ \\
\hline $\begin{array}{l}\text { In sagittal } \\
\text { plane }\end{array}$ & $\begin{array}{l}\text { Slight hyperlordotic posture } \\
\text { - } \text { Head and neck slightly projected forwards; } \\
\text { - } \text { Cervical arrow } 3.2 \mathrm{~cm} \text {; } \\
\text { - } \quad \text { Lumbar arrow } 4.8 \mathrm{~cm} \text {; } \\
\text { - } \quad \text { Slightly anteverted pelvis. }\end{array}$ & $\begin{array}{l}\text { We recommend } \\
\text { abdominal muscles } \\
\text { toning and dorsal } \\
\text { muscles toning, } \\
\text { postural self-control }\end{array}$ \\
\hline $\begin{array}{l}\text { S.M. } \\
\text { In frontal } \\
\text { plane }\end{array}$ & $\begin{array}{l}\text { Levoconvex dorsolumbar scoliotic posture } \\
\text { - head leftwards, asymmetric scapulae and shoulders, } \\
\text { lower on the right side by } 4^{\circ} \\
\text { - Uneven waist triangles, larger and sharper on the right; } \\
\text { - Asymmetric pelvis, higher gluteal fold and iliac crest on } \\
\text { the right side by } 3^{\circ} \text {. }\end{array}$ & $\begin{array}{l}\text { We recommend } \\
\text { kinesiotherapy, } \\
\text { orthopaedic and } \\
\text { radiology consult }\end{array}$ \\
\hline
\end{tabular}


No. 2, Vol. XVII /2016

\begin{tabular}{|c|c|c|}
\hline $\begin{array}{l}\text { In sagittal } \\
\text { plane }\end{array}$ & $\begin{array}{l}\text { Hyperlordotic posture } \\
\text { - } \quad \text { Cervical arrow } 2.3 \mathrm{~cm} ; \\
\text { - } \quad \text { Lumbar arrow } 5.1 \mathrm{~cm} ; \\
\text { - } \quad \text { Slightly anteverted pelvis. } \\
\end{array}$ & $\begin{array}{l}\text { We recommend } \\
\text { kinesiotherapy }\end{array}$ \\
\hline $\begin{array}{l}\text { M.S. } \\
\text { In frontal } \\
\text { plane }\end{array}$ & $\begin{array}{l}\text { Slight levoconvex dorsolumbar scoliotic posture } \\
\text { - asymmetric scapulae and shoulders, lower on the right } \\
\text { by between } 1 \text { and } 4^{\circ} \text {; } \\
\text { - Uneven waist triangles, larger and sharper on the right; } \\
\text { - Asymmetric pelvis. }\end{array}$ & $\begin{array}{l}\text { We recommend } \\
\text { posture correction } \\
\text { through } \\
\text { kinesiotherapy }\end{array}$ \\
\hline $\begin{array}{l}\text { In sagittal } \\
\text { plane }\end{array}$ & $\begin{array}{l}\text { Hyperlordotic posture } \\
\text { - } \quad \text { Cervical arrow } 2.4 \mathrm{~cm} ; \\
\text { - } \text { Lumbar arrow } 3.1 \mathrm{~cm} ; \\
\text { - } \text { anteverted pelvis; } \\
\text { - } \text { Barycentre deviation towards the back, greater support } \\
\text { on the heels. }\end{array}$ & $\begin{array}{l}\text { We recommend } \\
\text { abdominal muscles } \\
\text { toning and dorsal } \\
\text { muscles toning }\end{array}$ \\
\hline $\begin{array}{l}\text { I.G. } \\
\text { In frontal } \\
\text { plane }\end{array}$ & $\begin{array}{l}\text { Levoconvex dorsolumbar scoliotic posture } \\
\text { - asymmetric scapulae and shoulders, the right lower by } \\
\text { around } 3-4^{\circ} \text {; } \\
\text { - asymmetric waist triangles, the right sharper and more } \\
\text { pronounced; } \\
\text { - asymmetric pelvis in this plane, higher by } 2-3^{\circ} \text { on the } \\
\text { right. }\end{array}$ & $\begin{array}{l}\text { We recommend } \\
\text { kinesiotherapy, } \\
\text { orthopaedic and } \\
\text { radiology } \\
\text { evaluation, } \\
\text { assessment of lower } \\
\text { limbs length } \\
\end{array}$ \\
\hline $\begin{array}{l}\text { In sagittal } \\
\text { plane }\end{array}$ & $\begin{array}{l}\text { Hyperlordotic posture } \\
\text { - } \text { Head and neck slightly projected forwards, shoulders } \\
\text { forwards; } \\
\text { - Cervical arrow } 1.8 \mathrm{~cm} \text {; } \\
\text { - } \quad \text { Lumbar arrow } 4.3 \mathrm{~cm} \text {; } \\
\text { - } \text { anteverted pelvis; } \\
\text { - } \quad \text { Slight barycentre deviation forwards, with greater } \\
\text { support on the front leg }\end{array}$ & $\begin{array}{l}\text { We recommend } \\
\text { kinesiotherapy }\end{array}$ \\
\hline $\begin{array}{l}\text { E.A. } \\
\text { In sagittal } \\
\text { plane }\end{array}$ & $\begin{array}{l}\text { Kypholordotic posture } \\
\text { - } \quad \text { Head and neck projected forwards, shoulders forwards; } \\
\text { - } \quad \text { Cervical arrow } 3.5 \mathrm{~cm} \text {; } \\
\text { - } \quad \text { Lumbar arrow } 5.6 \mathrm{~cm} \text {; } \\
\text { - } \text { anteverted pelvis. }\end{array}$ & $\begin{array}{l}\text { We recommend } \\
\text { kinesiotherapy to } \\
\text { correct posture and } \\
\text { abdominal muscles } \\
\text { and back muscles } \\
\text { toning }\end{array}$ \\
\hline $\begin{array}{l}\text { B.B. } \\
\text { In sagittal } \\
\text { plane }\end{array}$ & $\begin{array}{l}\text { Hyperlordotic posture } \\
\text { - } \text { Head and neck slightly projected forwards, shoulders } \\
\text { forwards; } \\
\text { - } \text { Cervical arrow } 1.8 \mathrm{~cm} \text {; } \\
\text { - } \text { Lumbar arrow } 4.4 \mathrm{~cm} \text {; } \\
\text { - } \text { slightly anteverted pelvis. }\end{array}$ & $\begin{array}{l}\text { We recommend } \\
\text { kinesiotherapy to } \\
\text { correct posture, } \\
\text { abdominal muscles } \\
\text { toning, head } \\
\text { position correction }\end{array}$ \\
\hline
\end{tabular}

\section{Conclusions}

In summary, and with the above limitations in mind, the authors advocate the following recommendations to reduce the risk of injury in Rhythmic Gymnastics: 1. Stretch a minimum of 40 minutes per day. 2. Limit conditioning 
to a maximum of 6 hours per week. This may reduce likelihood of fracture. 3 . Limit training to a maximum of 30 hours per week, which may reduce likelihood of fracture; a further restriction of training to a maximum of 20 hours per week may reduce muscle-tendon unit injuries. 4. Measures to reduce the prevalence of low-back pain and knee pain should be sought. For example, a prophylactic regimen of abdominal strength exercises, antilordotic exercises, vastus medialis obliquus strength exercises, and quadriceps stretches could be incorporated into training. Reduction of the number of repetitions of back hyperextension techniques could be considered. (Zetaruk et al., 2006, p. 100)

The Tanchev et al. study generates an etiologic hypothesis including the "dangerous triad" of generalized joint laxity, delayed maturity, and asymmetric overloading of the spine. (Tanchev, 2000, p. 1367)

As a general conclusion, we highlight the need to implement individual programs for the rehabilitation or correction of faulty postures, to be performed following specific trainings. We can also introduce a series of drills during trainings, drills meant to correct such attitudes and to reduce the number of repetitions, which actually deepen these deficiencies.

\section{References}

1. BENOIST, M. (2003). Natural history of the aging spine, European Spine Journal, 2 (12), 86-89;

2. D'HEMECOURT, P.A. (2012). Spinal Deformity in young athletes, Clinical Sports Medicine, 3 (31), 25-35;

3. STOŠIĆ, D., MILENKOVIĆ, S., ŽIVKOVIĆ, D. (2011). The influence of sport on the development of postural disorders in athlete, Facta Universitatis, Series: Physical Education and Sport, 4 (9), Special Issue, 375-384;

4. MKAOUER B., AMARA S., TABKA Z. (2012). Split leap with and without ball performance factors in rhythmic gymnastics, Science of gymnastics Journal, 2 (4), 75-81;

5. BROOKS, K. (2011). Muscle Used in Rhythmic Gymnastics, Livestrong;

6. BROOKS, TJ. (2003). Women's collegiate gymnastics: A multifactorial approach to training and conditioning, Strength Conditioning Journal, (25), 23-37;

7. DI CAGNO A., BALDARI C., BATTAGLIA C., GALLOTTA M.C., VIDEIRA M., PIAZZA M., GUIDETTI L. (2010). Preexercise static stretching effect on leaping performance in elite rhythmic gymnasts. Journal of Strength and Conditioning Research, 8 (24), 1995-2000;

8. KRUSE, D. \& LEMMEN, B. (2009). Spine injuries in the sport of gymnastics. Current Sports Medicine Reports, 1 (8), 20-28;

9. HUTCHINSON, MR. (1999). Low back pain in elite rhythmic gymnasts, Medicine Science Sports Exercises, (31), 1686;

10. DONATELLI, R., DIMOND, D. (2012). Sport-specific biomechanics of spinal injuries in the athlete (throwing athletes, rotational sports, and contact-collision sports), Clinical Sports Medicine, 3 (31), 12;

11. ZETARUK, M., VIOLAN, M., ZURAKOWSKI, D., MITCHELL W., JR. 
\& MICHELI L. (2006). Injuries and training recommendations in elite rhythmic gymnastics, Medicina de L'esport, (151), 100-106;

12. TANCHEV, P., DZHEROV, A., PARUSHEV, A., DIKOV, D., AND TODOROV, M. (2000). Scoliosis in Rhythmic Gymnasts, SPINE, 11 (25), Lippincott Williams \& Wilkins, Inc.,1367-1372.

\title{
ASPECTE PRIVIND ATITUDINILE VICIOASE ALE COLOANEI ÎN GIMNASTICA RITMICĂ FEMININĂ
}

\author{
Moraru Cristina - Elena ${ }^{1}$ \\ ${ }^{1}$ Universitatea "Alexandru Ioan Cuza" Iasi, Str. Carol Street 11, 700506, Romania
}

Cuvinte cheie: atitudini deficiente, postură, gimnastică ritmică

\section{Rezumat}

Scopul acestei lucrări a fost acela de a observa dacă în rândul copiilor care practică gimnastica ritmica de performanţă apar atitudini lordotice sau scoliotice. Identificarea timpurie a atitudinilor vicioase la sportivele care practica gimnastica ritmică ne poate conduce spre iniţierea unui program de recuperare pentru a preveni dezvoltarea acestora. În cadrul acestei cercetări au fost analizate 10 gimnaste de categoria a IV-a cu vârsta cuprinsă între 8-10 ani. Măsurătorile au fost efectuate cu ajutorul platformei stabilometrice GPS 400 - Global Postural System. În urma măsurătorilor efectuate am depistat un număr de 10 gimnaste cu atitudini lordotice si opt gimnaste cu atitudini scoliotice. Ca o concluzie generală, putem sublinia ideea necesităţii implementării unor programe individuale de recuperare/corectare a atitudinilor deficiente, care să fie efectuate după antrenamentele specifice.

\section{Introducere}

Coloana vertebrală este flexibilă și are mai multe elemente în componență. Rolul său funcțional este de a menține stabilitatea și poziția dreaptă, precum și de a oferi mobilitate segmentelor. Coloana vertebrală cuprinde un element static, imobil (vertebrele) şi un element flexibil și mobil (complexul de trei încheieturi, care include discul intervertebral și două încheieturi posterioare). Mișcarea coloanei vertebrale, stabilitatea, echilibrul și controlul postural sunt în sarcina acțiunii antagoniste a puternicelor grupe de mușchi flexori și extensori. (Benoist, 2003, p.86)

Diformitățile coloanei reprezintă un spectru de maladii adesea cu debut în copilărie sau adolescență, cu posibile ramificații ale durerii și diformității în viața adultă. (D'Hemecourt, 2012, p. 25).

Incidența crescută a problemelor posturale în anumite ramuri sportive, în special în rândul adolescenților, a ridicat problema influenței activităţilor sportive asupra posturii sportivilor. 
Deviațiile de la postura corectă, ca rezultat al unui sport specific sau al suprasolicitărilor de la antrenamente, au fost dovedite în lucrările a numeroși autori (Grabara \& Hadzik, 2009; Slawinska, Rozek \& Ignasiak, 2006).

Unele probleme posturale sunt mai des întâlnite în anumite ramuri sportive, drept pentru care se presupune că cerințele specifice sportului și solicitările care apar în timpul executării elementelor tehnice, precum și repetarea prelungită a elementelor respective influențează evoluția acestor probleme posturale. Anumite sporturi în care sexul feminin este predominant, cum ar fi gimnastica ritmică, dansul și patinajul artistic se caracterizează printro mare amplitudine a mișcării în zona coloanei vertebrale (Cirillo \& Jackson, 1985; Micheli 1983, Sward, Hellstrom \&i Jacobsonn, 1990). Sportivii care practică asemenea sporturi sunt mai vulnerabili la accidentări la nivelul coloanei vertebrale și la dezvoltarea anormală a acesteia.

Practic nu există în ziua de azi vreun sport care să nu cauzeze probleme ale coloanei, dar nivelul și tipul acestor probleme depind de natura sportului respectiv. (Stošić et al., 2011, p. 375)

Capacitatea de executare este foarte complexă în gimnastica ritmică, pentru că gimnaștii se confruntă adesea cu situații în care au roluri duale: sărituri, întoarceri sau echilibru în menținerea sau mișcarea obiectului.

Așadar, nu putem ține cont doar de aspectul cantitativ, ci și de calitatea execuției, care depinde în mod direct de nivelul de coordonare, de măiestrie tehnică și de performanță fizică a gimnaștilor (Mkaouer et al., 2012, p.75).

Pentru a combina mai multe elemente, gimnaștii au nevoie de un nivel proprioceptiv și de control motor ridicat, care se obține prin dezvoltare neurologică, ce necesită ani întregi de exercițiu. Sistemul neuromuscular trebuie să coordoneze mișcările, deci este nevoie de un control foarte specific. (Brooks, 2011).

Practicanții gimnasticii ritmice au nevoie de o flexibilitate foarte bună și de forță pentru a atinge și a stăpâni foarte rapid săriturile cu amplitudine mare cerute de Codul Internațional de Punctaj (Brooks, 2003, p. 23; Di Cagno, 2010, p. 1995). Mai multe poziții corporale cerute în gimnastică prezintă riscuri de accidentări la coloană. Practicanții de gimnastică ritmică se antrenează și concurează la mai multe probe. În general, se pune presiune pe coloana vertebrală prin dobândirea abilităților de flexie și extensie extreme ale coloanei vertebrale, niveluri care nu se mai întâlnesc în alte sporturi.

Chiar și un ochi neavizat va observa că gimnastica cere un nivel de flexibilitate, condiție fizică și formă sportivă foarte rar întâlnite în alte sporturi. (Kruse \&, Lemmen, 2009, p. 20)

Foarte mulți gimnaști se plâng de dureri în zona lombară; numeroase studii epidemiologice și sinteze au arătat că procentajele se situează undeva $25 \%$ și $85 \%$. În ceea ce privește practicanții de gimnastică ritmică, un studiu publicat în 1999 a arătat că $86 \%$ dinte gimnaștii din cadrul studiului s-au plâns de dureri lombare (16). (Hutchinson, 1999, p. 1686). 


\section{Material şi metodă}

Elaborarea unei scheme eficiente de tratament depinde de examinarea minuțioasă a aparatelor musculoscheletal și neuromuscular, care mențin stabilitatea coloanei; trebuie să urmeze apoi un tratament eficient care include exerciţii terapeutice pentru a corecta deficiențele care pot fi depistate. Orice program de recuperare detaliat ar trebui să includă corectarea dezechilibrelor musculare rezultate din lipsuri la nivel de flexibilitate, forță, rezistență și echilibru. (Donatelli \& Dimond, 2012, p. 12).

Anumite sporturi în care sexul feminin este predominant, cum ar fi gimnastica ritmică, dansul și patinajul artistic se caracterizează printr-o mare amplitudine a mișcării în zona coloanei vertebrale, pentru că această regiune este solicitată intens. Unele probleme posturale sunt mai des întâlnite în anumite domenii sportive, motiv pentru care se presupune că cerințele specifice sportului și solicitările implicate de executarea anumitor elemente tehnice și de repetarea continuă a acestor elemente influențează de fapt evoluția atitudinilor vicioase. Sportivii care practică astfel de sporturi sunt mai vulnerabili la accidentări la nivelul coloanei vertebrale și la dezvoltarea anormală a acesteia.

Scopul acestei lucrări a fost acela de a observa dacă în rândul copiilor care practică gimnastica ritmica de performanţă apar atitudini lordotice sau scoliotice.

Ipoteza lucrării. Identificarea timpurie a atitudinilor vicioase la sportivele care practica gimnastica ritmica ne poate conduce spre iniţierea unui program de recuperare pentru a preveni dezvoltarea acestora.

În cadrul acestei cercetări au fost analizate 10 gimnaste de categoria a IVa cu vârsta cuprinsă între 8-10 ani. Măsurătorile au fost efectuate cu ajutorul platformei stabilometrice GPS 400 - Global Postural System.

\section{Rezultate şi discuţii}

În urma măsurătorilor efectuate am depistat un număr de 10 gimnaste cu atitudini lordotice si opt gimnaste cu atitudini scoliotice.

Table 1 Diagnostic funcţional a practicantelor de gimnastică ritmică

\begin{tabular}{|c|c|c|}
\hline $\begin{array}{c}\text { N.P/ } \\
\text { Deviaţie }\end{array}$ & Diagnostic functional & Recomandări \\
\hline $\begin{array}{l}\text { V.L. } \\
\text { In plan } \\
\text { frontal }\end{array}$ & $\begin{array}{l}\text { Atitudine scoliotica dorsolombară dextroconvexă } \\
\text { - asimetrie a umerilor şi scapulelor, partea stângă mai } \\
\text { coborâtă cu } 4^{\circ} \text {; } \\
\text { - bazin asimetric mai ascesionat cu } 3^{\circ} \text { de partea stângă; } \\
\text { - triunghiuri ale taliei inegale mai accentuate de partea } \\
\text { stângă. }\end{array}$ & $\begin{array}{l}\text { Se recomandă } \\
\text { kinetoterapie, consult } \\
\text { ortopedic şi } \\
\text { radiografie coloana } \\
\text { vertebrală }\end{array}$ \\
\hline $\begin{array}{l}\text { În plan } \\
\text { sagital }\end{array}$ & $\begin{array}{l}\text { Atitudine hiperlordotică cu raza mare de curbură } \\
\text { - cap şi gât uşor proiectat spre înainte; } \\
\text { - } \quad \text { săgeata cervicală } 1.1 \mathrm{~cm} ;\end{array}$ & $\begin{array}{l}\text { Se recomandă } \\
\text { kinetoterapie, consult } \\
\text { ortopedic şi }\end{array}$ \\
\hline
\end{tabular}




\begin{tabular}{|c|c|c|}
\hline & $\begin{array}{l}\text { - } \text { săgeata lombară } 3.4 \mathrm{~cm} \text {; } \\
\text { - } \text { deviaţie a centrului de greutate spre anterior cu sprijin } \\
\text { mai mare pe antepicior şi bazin anteversat. }\end{array}$ & radiologic \\
\hline $\begin{array}{l}\text { A.R. } \\
\text { În plan } \\
\text { frontal }\end{array}$ & $\begin{array}{l}\text { Uşoară tendinţă de atitudine scoliotică dorso-lombaăa } \\
\text { dextroconvexă } \\
\text { - } \quad \text { umeri asimetrici, stângul mai coborât cu } 3^{\circ} \text {; } \\
\text { - } \quad \text { scapule asimetrice, stânga mai coborâtă cu } 3^{\circ} \text {; } \\
\text { - } \quad \text { triunghi al taliei de partea stângă uşor mai accentuat; } \\
\text { - } \quad \text { pozitie incorectă a capului (răsucit spre stânga şi } \\
\text { înclinat spre dreapta). }\end{array}$ & $\begin{array}{l}\text { Se recomandă } \\
\text { autocontrol postural, } \\
\text { corectarea activă a } \\
\text { posturii, tonifierea } \\
\text { musculaturii spatelui. }\end{array}$ \\
\hline $\begin{array}{l}\text { In plan } \\
\text { sagital }\end{array}$ & $\begin{array}{l}\text { Atitudine hiperlordotică } \\
\text { - } \quad \text { cap şi gât uşor proiectate spre înainte; } \\
\text { - } \quad \text { săgeata cervicală } 1.3 \mathrm{~cm} \text {; } \\
\text { - } \quad \text { săgeata lombară } 3.4 \mathrm{~cm} \text {; } \\
\text { - } \quad \text { bazin anteversat, genunchi în uşoară hiperextensie. }\end{array}$ & $\begin{array}{l}\text { Se recomandă } \\
\text { kinetoterapie în } \\
\text { vederea corectării } \\
\text { posturii, tonifierea } \\
\text { musculaturii } \\
\text { abdominale şi } \\
\text { corectarea poziţiei } \\
\text { bazinului. }\end{array}$ \\
\hline $\begin{array}{l}\text { A.D. } \\
\text { In plan } \\
\text { frontal }\end{array}$ & $\begin{array}{l}\text { Atitudine scoliotică uşoară la nivel dorsolombar } \\
\text { levoconvexă } \\
\text { - umeri asimetrici, dreptul mai coborât cu } 4^{\circ} \text {; } \\
\text { - distanţa lob ureche - articulaţia acromioclaviculară } \\
\text { mai mare cu } 2 \mathrm{~cm} \text { de partea dreaptă; } \\
\text { - triunghiuri ale taliei inegale, mai ascuţit şi mai mare de } \\
\text { partea dreaptă cu } 1,9 \mathrm{~cm} \text {; } \\
\text { - bazin uşor ascensionat cu } 1-2^{\circ} \text { de partea dreaptă. }\end{array}$ & $\begin{array}{l}\text { Se recomandă } \\
\text { kinetoterapie pentru } \\
\text { reeducarea posturii } \\
\text { corecte în acest plan. }\end{array}$ \\
\hline $\begin{array}{l}\text { In plan } \\
\text { sagital }\end{array}$ & $\begin{array}{l}\text { Rectitudine coloană cervicodorsală şi hiperlordoză } \\
\text { lombară înaltă } \\
\text { - } \quad \text { rectitudine a coloanei cervicale şi dorsale superioare; } \\
\text { - } \text { săgeata cervicală } 0.8 \mathrm{~cm} \text {; } \\
\text { - } \quad \text { săgeata lombară } 4 \mathrm{~cm} \text {; } \\
\text { - } \quad \text { exagerare a curburii lombare cu rază lungă cuprinzând } \\
\text { şi zona dorsală; }\end{array}$ & $\begin{array}{l}\text { Se recomandă } \\
\text { kinetoterapie în } \\
\text { vederea realinierii } \\
\text { posturale, corectarea } \\
\text { curburilor fiziologice, } \\
\text { control radiologic şi } \\
\text { ortopedic }\end{array}$ \\
\hline $\begin{array}{l}\text { L.M. } \\
\text { In plan } \\
\text { frontal }\end{array}$ & $\begin{array}{l}\text { Atitudine scoliotică dorsolombaraădextroconvexă } \\
\text { - } \text { asimetrie a umerilor şi scapulelor, mai coborâte de } \\
\text { partea stângă; } \\
\text { - } \text { triunghiuri ale taliei inegale, mai ascuţit de partea } \\
\text { stângă; } \\
\text { - } \quad \text { asimetrie a bazinului, mai ascensionat de partea stângă. }\end{array}$ & $\begin{array}{l}\text { Se recomandă } \\
\text { kinetoterapie }\end{array}$ \\
\hline $\begin{array}{l}\text { In plan } \\
\text { sagital }\end{array}$ & $\begin{array}{l}\text { Atitudine hiperlordotică } \\
\text { - } \quad \text { uşoara proiecție a capului şi gâtului spre înainte; } \\
\text { - } \text { săgeata cervicală } 2.4 \mathrm{~cm} \text {; } \\
\text { - } \text { săgeata lombară } 4.4 \mathrm{~cm} ; \\
\text { - } \text { bazin anteversat. }\end{array}$ & $\begin{array}{l}\text { Se recomandă } \\
\text { tonifierea } \\
\text { musculaturii } \\
\text { abdominale şi } \\
\text { musculaturii spatelui } \\
\text { la nivel dorsal } \\
\end{array}$ \\
\hline $\begin{array}{l}\text { F.D. } \\
\text { In plan }\end{array}$ & $\begin{array}{l}\text { Atitudine scoliotică dorsal levoconvexă şi lombar } \\
\text { dextroconvexă }\end{array}$ & $\begin{array}{l}\text { Se recomandă } \\
\text { corectarea posturii }\end{array}$ \\
\hline
\end{tabular}




\begin{tabular}{|c|c|c|}
\hline frontal & $\begin{array}{l}\text { - } \text { asimetrie a umerilor şi scapulelor, partea dreaptă mai } \\
\text { coborată cu } 2^{\circ} \text {; } \\
\text { - } \text { triunghiuri ale taliei asimetrice atât ca forma cât şi ca } \\
\text { mărime, mai ascuţit de partea stângă şi uşor mai mare de } \\
\text { partea dreaptă; } \\
\text { - bazin uşor asimetric mai ascensionat de partea stângă. }\end{array}$ & prin kinetoterapie \\
\hline $\begin{array}{l}\text { In plan } \\
\text { sagital }\end{array}$ & $\begin{array}{l}\text { Uşoară atitutudine hiperlordotică } \\
\text { - } \quad \text { uşoară proiecţie a capului şi umerilor spre înainte; } \\
\text { - } \text { săgeata cervicală } 3.2 \mathrm{~cm} \text {; } \\
\text { - } \quad \text { săgeata lombară } 4.8 \mathrm{~cm} ; \\
\text { - } \quad \text { bazin uşor anteversat. }\end{array}$ & $\begin{array}{l}\text { Se recomandă } \\
\text { tonifierea } \\
\text { musculaturii } \\
\text { abdominale şi a } \\
\text { musculaturii spatelui } \\
\text { la nivel dorsal, } \\
\text { autocontrol postural }\end{array}$ \\
\hline $\begin{array}{l}\text { S.M. } \\
\text { In plan } \\
\text { frontal }\end{array}$ & $\begin{array}{l}\text { Atitudine scoliotică dorsolombară levoconvexă } \\
\text { - cap răsucit spre stânga, umeri şi scapule asimetrice, } \\
\text { mai coborată de partea dreaptă cu } 4^{\circ} \text {; } \\
\text { - triunghiuri ale taliei inegale, mai mare şi mai ascuţit de } \\
\text { partea dreaptă; } \\
\text { - asimetrie a bazinului cu ascensionarea pliului subfesier } \\
\text { şi a crestei iliace de partea dreaptă cu } 3^{\circ} \text {. }\end{array}$ & $\begin{array}{l}\text { Se recomandă } \\
\text { kinetoterapie, } \\
\text { control radiologic şi } \\
\text { medic ortoped }\end{array}$ \\
\hline $\begin{array}{l}\text { In plan } \\
\text { sagital }\end{array}$ & $\begin{array}{l}\text { Atitudine hiperlordotică } \\
\text { - săgeata cervicală } 2.3 \mathrm{~cm} ; \\
\text { - săgeata lombară } 5.1 \mathrm{~cm} \\
\text { - bazin uşor anteversat. }\end{array}$ & $\begin{array}{l}\text { Se recomandă } \\
\text { kinetoterapie }\end{array}$ \\
\hline $\begin{array}{l}\text { M.S. } \\
\text { In plan } \\
\text { frontal }\end{array}$ & $\begin{array}{l}\text { Uşoară atitudine scoliotică dorsolombară levoconvexă } \\
\text { - asimetrie a umerilor şi scapulelor, mai coborâte de } \\
\text { partea dreaptă între } 1 \text { şi } 4^{\circ} \text {; } \\
\text { - } \text { triunghiuri ale taliei inegale, mai mare şi mai ascuţit de } \\
\text { partea dreaptă; } \\
\text { - bazin asimetric. }\end{array}$ & $\begin{array}{l}\text { Se recomandă } \\
\text { corectarea posturii } \\
\text { prin kinetoterapie }\end{array}$ \\
\hline $\begin{array}{l}\text { In plan } \\
\text { sagital }\end{array}$ & $\begin{array}{l}\text { Atitudine hiperlordotică } \\
\text { - } \quad \text { săgeata cervicală } 2.4 \mathrm{~cm} ; \\
\text { - } \text { săgeata lombară } 3.1 \mathrm{~cm} ; \\
\text { - } \text { bazin anteversat; } \\
\text { - } \quad \text { deplasare a centrului de greutate spre posterior cu } \\
\text { sprijin mai mare pe călcâie. }\end{array}$ & $\begin{array}{l}\text { Se recomandă } \\
\text { tonifierea } \\
\text { musculaturii } \\
\text { abdominale şi a } \\
\text { musculaturii spatelui } \\
\text { la nivel dorsal }\end{array}$ \\
\hline $\begin{array}{l}\text { I.G. } \\
\text { In plan } \\
\text { frontal }\end{array}$ & $\begin{array}{l}\text { Atitudine scoliotică dorsolombară levoconvexă } \\
\text { - asimetrie a umerilor şi scapulelor, partea dreaptă mai } \\
\text { coborâtă cu aproximativ } 3-4^{\circ} \text {; } \\
\text { - } \text { triunghiuri ale taliei asimetrice, dreptul mai accentuat } \\
\text { şi mai ascuţit; } \\
\text { - bazin asimetric în acest plan, mai ascensionat de partea } \\
\text { dreaptă cu } 2-3^{\circ} \text {. }\end{array}$ & $\begin{array}{l}\text { Se recomandă } \\
\text { kinetoterapie, } \\
\text { evaluare ortopedică } \\
\text { şi radiologică, } \\
\text { evaluarea lungimii } \\
\text { membrelor inferioare }\end{array}$ \\
\hline $\begin{array}{l}\text { In plan } \\
\text { sagital }\end{array}$ & $\begin{array}{l}\text { Atitutdine hiperlordotică } \\
\text { - } \quad \text { uşoară proiecţie a capului şi gâtului spre înainte, } \\
\text { umerii adduşi spre înainte; } \\
\text { - } \quad \text { săgeata cervicală } 1.8 \mathrm{~cm} \text {; } \\
\text { - } \text { săgeata lombară } 4.3 \mathrm{~cm} ;\end{array}$ & $\begin{array}{l}\text { Se recomandă } \\
\text { kinetoterapie }\end{array}$ \\
\hline
\end{tabular}




\begin{tabular}{|c|c|c|}
\hline & $\begin{array}{l}\text { - } \quad \text { bazin anteversat; } \\
\text { - } \quad \text { tendinţă de deplasare anterioară a centrului de greutate } \\
\text { cu sprijin preponderent pe antepicior. }\end{array}$ & \\
\hline $\begin{array}{l}\text { E.A. } \\
\text { In plan } \\
\text { sagital }\end{array}$ & $\begin{array}{l}\text { Atitudine cifolordotica } \\
\text { - cap şi gât proiectate anterior, umeri uşor adduşi spre } \\
\text { înainte; } \\
\text { - } \text { săgeata cervicală } 3.5 \mathrm{~cm} \text {; } \\
\text { - } \text { săgeata lombară } 5.6 \mathrm{~cm} \text {; } \\
\text { - } \text { bazin anteversat. }\end{array}$ & $\begin{array}{l}\text { Se recomandă } \\
\text { kinetoterapie în } \\
\text { vederea corectarii } \\
\text { posturii şi tonifierea } \\
\text { musculaturii } \\
\text { abdominale şi a } \\
\text { spatelui }\end{array}$ \\
\hline $\begin{array}{l}\text { B.B. } \\
\text { In plan } \\
\text { sagital }\end{array}$ & $\begin{array}{l}\text { Atitudine hiperlordotică } \\
\text { - cap şi gât aplecat şi uşor proiectat spre înainte, umeri } \\
\text { uşor adduşi spre înainte; } \\
\text { - } \text { săgeata cervicală } 1.8 \mathrm{~cm} \text {; } \\
\text { - } \quad \text { săgeata lombară } 4.4 \mathrm{~cm} ; \\
\text { - } \text { bazin uşor anteversat. }\end{array}$ & $\begin{array}{l}\text { Se recomandă } \\
\text { kinetoterapie în } \\
\text { vederea corectării } \\
\text { posturii, tonifierii } \\
\text { muşchilor } \\
\text { abdominali, } \\
\text { corectarea poziţiei } \\
\text { capului }\end{array}$ \\
\hline
\end{tabular}

\section{Concluzii}

Pe scurt, ținând cont de toate limitele studiului, autorii au următoarele recomandări pentru a reduce riscul de accidentări în gimnastica ritmică: 1 . Utilizarea exerciţiilor de tip stretching cel puțin 40 de minute pe zi. 2 . Reducerea antrenamentelor specifice la 6 ore pe săptămână, lucru care va reduce riscul de fracturi. 3. Reducerea antrenamentelor la maxim 30 de ore pe săptămână, lucru care va reduce riscul de fracturi; dacă se vor reduce antrenamentele și mai mult, la maxim 20 de ore pe săptămână, veți reduce riscul de accidentări la nivelul tendoanelor. 4. Trebuie luate în calcul și măsuri de reducere a durerilor lombare și de genunchi. Un asemenea regim profilactic ar trebui să includă exerciții pentru mușchii abdominali, antilordotice, pentru întărire a mușchiului vastus medialis obliquus, precum și stretching la nivelul cvadricepșilor. Ar mai trebui luată în considerare reducerea numărului de repetări pentru tehnicile de hiperextensii la nivelul spatelui. (Zetaruk et al., 2006, p.100).

Studiul lui Tanchev et al. include o ipoteză etiologică bazată pe ,triada periculoasă" formată din laxitatea generalizată a încheieturilor, întârzierea maturizării și solicitările asimetrice la nivelul coloanei vertebrale. (Tanchev, 2000, p. 1367).

Ca o concluzie generală, putem sublinia ideea necesităţii implementării unor programe individuale de recuperare/corectare a atitudinilor deficiente, care să fie efectuate după antrenamentele specifice. De asemenea, pot fi introduse o serie de exerciţii în timpul antrenamentelor care să ajute la corectarea atitudinilor şi reducerea numărului de repetări a exerciţiilor care accentuează aceste deficienţe. 